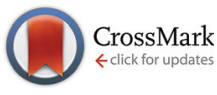

Cite this: Dalton Trans., 2016, 45 18443

Received 11th October 2016, Accepted 21st October 2016

DOI: $10.1039 / c 6 d t 03930 h$

\section{Direct synthesis of non-breathing MIL-53(Al)(ht) from a terephthalate-based ionic liquid as linker precursor $\dagger$}

\author{
Marcus Fischer, ${ }^{a}$ Johannes Schwegler, ${ }^{b}$ Carolin Paula, ${ }^{a}$ Peter S. Schulz ${ }^{\star b}$ and \\ Martin Hartmann*a
}

www.rsc.org/dalton

An organic imidazolium salt of terephthalic acid has been utilized as a linker precursor for the synthesis of an aluminum-based metal organic framework (MOF) with MIL-53(ht) structure. This material shows the predicted porosity in terms of nitrogen and hydrogen sorption without exhibiting the breathing effect typical for MIL-53 (Al) materials.

While the formation processes of many MOFs still remains a hot topic of ongoing research, irreproducible syntheses resulting in altering material properties often prevent their commercialization. To overcome this hurdle, contribute to the overall understanding of the formation process, and find benign, mild routes for the selective syntheses of the targeted frameworks, many variations of classical hydrothermal or solvothermal MOF syntheses have been reported in recent years. These include electrochemical, ambient pressure, and modulated procedures. ${ }^{1}$ In modulated syntheses, ${ }^{2}$ a soluble complex of the metal cations and ligands, with coordinating moieties similar to those of the MOF linker, is performed in situ during the synthesis. Furthermore, a controlled SBU (secondary building unit) approach has been developed. Here, multinuclear complexes are formed in a preliminary step and used directly as nodes to build up the framework by ligand exchange. ${ }^{3}$ However, with respect to the linker, relatively few studies are reported using precursors other than the free carboxylic acid species. A notable exception is the trimethylester of trimesic acid employed in the hydrothermal synthesis of MIL-100(Al). ${ }^{4}$

Recently, a new environmentally friendly synthesis of MIL-53(Al) was reported by Liu et al. ${ }^{5}$ In this ionothermal approach, the ionic liquid 1-ethyl-3-methylimidazolium

\footnotetext{
${ }^{a}$ Erlangen Catalysis Resource Center, Friedrich-Alexander Universität Erlangen-Nürnberg, Egerlandstr. 3, 91058 Erlangen, Germany. E-mail: Martin.Hartmann@fau.de; Fax: +49 9131 8567401; Tel: +49 91318528792 ${ }^{b}$ Institute of Chemical Reaction Engineering, Friedrich-Alexander Universität Erlangen-Nürnberg, Egerlandstr. 3, 91058 Erlangen, Germany.

E-mail: Peter.Schulz@fau.de; Fax:+49 9131 8527421; Tel: +49 91318527431

$\dagger$ Electronic supplementary information (ESI) available: Experimental details on linker precursor and materials syntheses, NMR spectra, elemental analyses, nitrogen sorption isotherms, SEM images. See DOI: 10.1039/c6dt03930h
}

bromide [EMIM]Br was used as a recyclable solvent. It results in a hydrophobic MIL-53 material, which is already in the htform. Using this procedure, the synthesis temperature could be reduced from $220^{\circ} \mathrm{C}$ to $150{ }^{\circ} \mathrm{C}$, but an even longer synthesis time (7 days) is required and subsequent calcination at $330^{\circ} \mathrm{C}$ is still mandatory to obtain a sufficiently crystalline material.

In this study, we utilized an ionic liquid, namely an imidazolium salt of terephthalic acid not only as reaction medium or structure directing agent ${ }^{6}$ but also as precursor for the organic linker. The salt 1-ethyl-3-methylimidazolium hydrogen terephthalate [EMIM][Hbdc] was chosen for the synthesis of MIL-53(Al) with the aim of enhancing the solubility and the availability of deprotonated terephthalate, thereby reducing the synthesis temperature and time, respectively.

[EMIM][Hbdc] is accessible via a modified procedure described by Massonne et al., ${ }^{7}$ where a $30 \%$ solution of 1-ethyl-3-methylimidazolium methylcarbonate in methanol is used as stable starting material and added dropwise to the prepared terephthalic acid (Scheme 1). After contact with the solid acid, immediate foaming can be observed and the acid is partially dissolved. The evolving gas is collected and measured in a volumetric cylinder, which allows easy monitoring of the reaction progress. Notably, the grade of deprotonation of the terephtalic acid can be controlled by adjusting the stoichiometric ratio. Full conversion of $1 \mathrm{eq}$. methylcarbonate to methanol and carbon dioxide can also be measured when only 0.5 eq. terephthalic acid is used. The product of this reaction, $[\mathrm{EMIM}]_{2}[\mathrm{bdc}]$, is completely soluble in the stoichiometrically produced amount of $\mathrm{MeOH}$. We propose that this species is also formed as an intermediate when 1 eq. $\mathrm{H}_{2}$ bdc is used, as

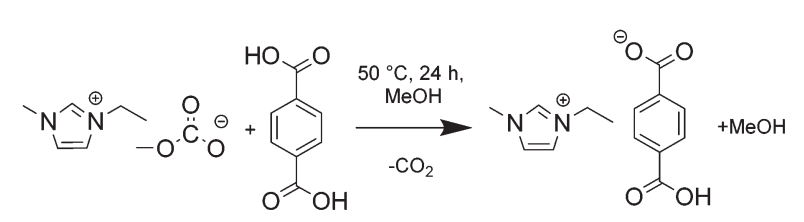

Scheme 1 Synthesis of [EMIM][Hbdc] from an [EMIM] methylcarbonate precursor. 

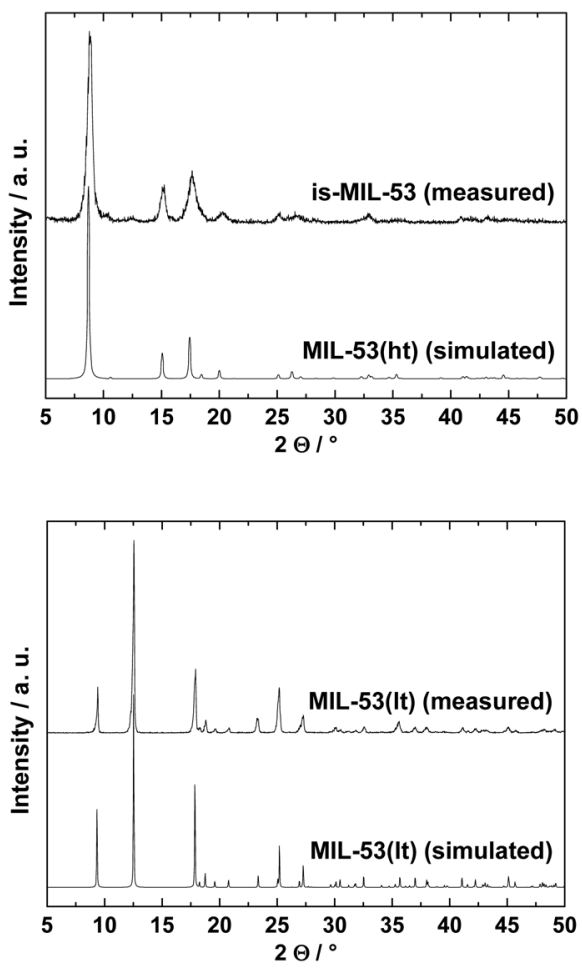

Fig. 1 Powder-XRD patterns of MIL-53(lt) and is-MIL-53.

this would be in agreement with the observed temporary dissolution. Upon further stirring $[\mathrm{EMIM}]_{2}[\mathrm{bdc}]$ reacts with the remaining 0.5 eq. $\mathrm{H}_{2}$ bdc to give [EMIM][Hbdc]. Due to its poor solubility in methanol, this species then precipitates from the solution.

The MIL-53 sample synthesized from [EMIM][Hbdc] (further denoted as is-MIL-53; is = imidazolium salt) at $130{ }^{\circ} \mathrm{C}$ was identified as pure ht-phase by its powder XRD pattern (Fig. 1). The porosity detected after drying at $130{ }^{\circ} \mathrm{C}$ is comparable to that of the hydrothermally synthesized sample of MIL-53, which was obtained following the literature procedure described in ref. 8 , after activation at $330{ }^{\circ} \mathrm{C}$. However, in case of is-MIL-53, the shapes of the nitrogen adsorption isotherm as well as the relatively broad reflexes of the XRD pattern indicate a high degree of imperfection of the crystallites.

The comparison of the textural properties of MIL-53 and is-MIL-53, calculated from nitrogen sorption isotherms at $-196{ }^{\circ} \mathrm{C}$ (Table 1), reveal that although the total pore volumes

Table 1 Specific surface areas and pore volumes of MIL-53 and is-MIL-53

\begin{tabular}{llll}
\hline & $S_{\mathrm{BET}}{ }^{a} / \mathrm{m}^{2} \mathrm{~g}^{-1}$ & $V_{\mathrm{p}, \text { total }}{ }^{b} / \mathrm{cm}^{3} \mathrm{~g}^{-1}$ & $V_{\mathrm{p}, \text { micro }}{ }^{c} / \mathrm{cm}^{3} \mathrm{~g}^{-1}$ \\
\hline MIL-53 & 1510 & 0.57 & 0.53 \\
is-MIL-53 & 1228 & 0.56 & 0.37
\end{tabular}

${ }^{a}$ Calculated in pressure ranges individually determined as proposed by Snurr et al. ${ }^{12}{ }^{b}$ Calculated at $p / p_{0}=0.6 .{ }^{c}$ Derived by the $t$-plot method. of the samples determined at a relative pressure of $p / p_{0}=0.6$ are almost identical, the micropore volume, calculated by the $t$-plot method, of is-MIL-53 is about $30 \%$ lower than the micropore volume of hydrothermally synthesized MIL-53. This discrepancy is not fully confirmed by the comparison of the BET surface areas, which is less than $20 \%$ lower for is-MIL-53. While the isotherm of MIL-53 reaches the saturation plateau at a very low relative pressure, typical for a purely microporous solid with uniform pore geometry, the isotherm of is-MIL-53 is constantly increasing with a significant rise at relative pressures above 0.7 . This rise at higher pressures can be assigned to early vapor condensation, initiated by either interparticle porosity or the presence of an additional meso- or macroporous compound. Both effects are conceivable when the morphology of the outer surface of the is-MIL-53 particles, as shown in the SEM image (Fig. S5 $\dagger$ ), is considered. While the MIL-53 particles have a totally plain surface and sharp edges, the surface of the is-MIL-53 crystallites is covered with an unregularly shaped phase which may comprise undefined mesopores and surely forms randomly sized interparticular macropores.

Interestingly, the powder X-ray diffraction pattern of isMIL-53 (Fig. 1) shows that the material is obtained in pure htphase directly from the synthesis without further activation at high temperature. This demonstrates a certain structure directing effect of the imidazolium cation since the conventional hydrothermal synthesis first of all results in the as-form of MIL-53, which contains a defined amount of 0.7 equivalents of unreacted terephthalic acid. This material is subsequently activated at $330{ }^{\circ} \mathrm{C}$ by thermal removal of the excess linker molecules and is then, after exposure to ambient air, present in the water loaded lt-form.

In contrast to the hydrothermally synthesized MIL-53, which is known for its breathing effect, viz. the temperature or sorbent induced phase transition between the narrow pore ltform (low temperature or sorbent $\left(\mathrm{H}_{2} \mathrm{O}\right)$ loaded) and the large pore ht-form (high temperature or sorbent free), the is-MIL-53 was only observed in the ht-form as evident from the powder XRD pattern (Fig. 1) and solid state ${ }^{27}$ Al-NMR spectra (Fig. 2),

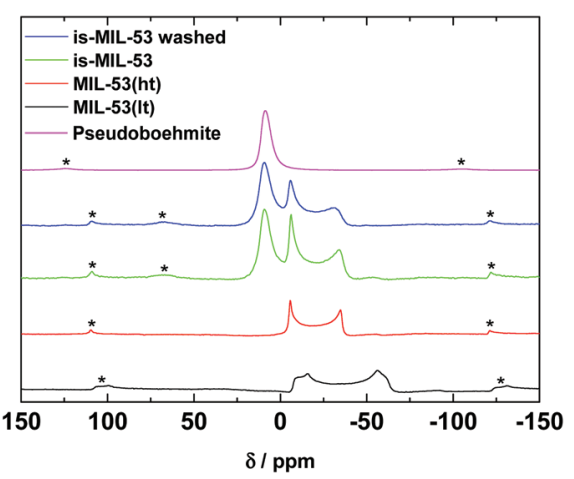

Fig. 2 Solid state ${ }^{27} \mathrm{Al}-\mathrm{MAS}$ NMR spectra of hydrothermally synthesized MIL-53 in It- and ht-form and [EMIM][Hbdc] derived is-MIL-53 after several days at ambient conditions and after washing with deionized water. Asterisks indicate spinning sidebands. 
Table 2 Parameters of the solid state ${ }^{27}$ Al-MAS NMR spectra of MIL-53

\begin{tabular}{llrll}
\hline & $\begin{array}{l}\delta_{\text {iso }} / \\
\text { ppm }\end{array}$ & $\begin{array}{l}C_{\mathrm{Q}} / \\
\mathrm{MHz}\end{array}$ & \multicolumn{1}{l}{$\begin{array}{l}\eta_{\mathrm{Q}} / \\
\text { MIL-53(lt) }\end{array}$} & $\begin{array}{l}\text { Lorentzian line } \\
\text { broadening/ppm }\end{array}$ \\
MIL-53(ht) & 4.02 & 10.70 & 0.12 & 0.81 \\
is-MIL-53 & 4.46 & 8.50 & 0.00 & 0.51 \\
is-MIL-53 ( $\mathrm{H}_{2} \mathrm{O}$ washed) & 4.15 & 8.55 & 0.00 & 0.52 \\
& 4.58 & 8.46 & 0.00 & 0.51
\end{tabular}

even after washing of the material with deionized water. That suggests, that the ht-form is stabilized either chemically, by reduced hydrophilicity, or by mechanical stabilization of the small crystallites due to incorporation into a matrix of extra framework alumina, preventing the phase transformation.

The latter suggestion is supported by the ${ }^{27} \mathrm{Al}-\mathrm{NMR}$ spectrum of is-MIL-53 (Fig. 2), which shows the characteristic signal of MIL-53(ht) while the presence of the lt-phase cannot be detected (Table 2). In addition, a second signal at $9.2 \mathrm{ppm}$ shows the presence of another octahedrally coordinated aluminum species. The obvious suspicion, that this is due to residual $\mathrm{Al}\left(\mathrm{NO}_{3}\right)_{3}$ can be disproved since the intensity of this peak is not effected by washing the sample with deionized water. The comparison of the SEM images of MIL-53 and isMIL-53 demonstrates, that the particles of the latter material possess a much rougher outer surface, indicating a shell of differently structured material. The most likely candidate being $\gamma-\mathrm{AlO}(\mathrm{OH})$ in the form of boehmite or pseudoboehmite (finely crystalline boehmite with a higher water content). From the measured shift of $8.7 \mathrm{ppm}$ for pseudoboehmite (Fig. 2), and the value of boehmite $(d=9.5 \mathrm{ppm})$ reported by Isobe et al. ${ }^{9}$ we conclude that the additional species is indeed $\gamma$-AlO $(\mathrm{OH})$. The elemental analysis (Table S2 $\dagger$ ) of is-MIL-53 supports this claim and suggests the presence of $c a .1$ eq. $\gamma$-AlO(OH). A similar phenomenon was found by Bezverkhyy et al. for MIL-53 after postsynthetic hydrothermal treatment. In their study, the shell material was also identified as $\gamma-\mathrm{AlO}(\mathrm{OH})$ which is formed at the surface after partial hydrolysis of the MOF. $^{10}$

Both MIL-53 samples, hydrothermally synthesized and isMIL-53, have been tested as potential candidates for the adsorptive storage of hydrogen at cryogenic temperatures $\left(-196{ }^{\circ} \mathrm{C}\right)$. The $\mathrm{H}_{2}$-isotherm of MIL-53 shows a large step in adsorption branch at approx. 10 bar, and a significant hysteresis (Fig. 3). This can be explained by the sorbent induced phase transformation, often referred to as breathing effect. After activation in vacuum at $170{ }^{\circ} \mathrm{C}, \mathrm{MIL}-53$ is present in the ht-form, as demonstrated by MAS-NMR-spectroscopy. By cooling to temperatures below $-100{ }^{\circ} \mathrm{C}$, it converts to the lt-form, even in absence of inducing sorbents. ${ }^{11}$ Upon adsorption of hydrogen the material is reconverted into the ht-form, but this process is very slow since the diffusion of the sorbent is highly restricted when the material is in the narrow-pore lt-state.

In contrast to this unusual behavior, is-MIL-53 shows a steadily rising hydrogen uptake, as expected for a rigid microporous solid. This is in full agreement with the results of

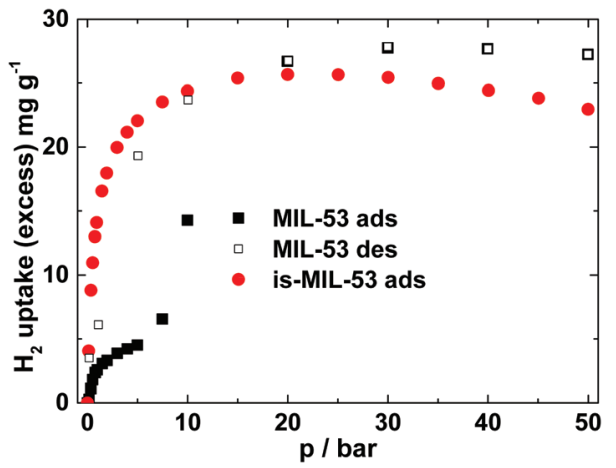

Fig. 3 Hydrogen sorption isotherms at $77 \mathrm{~K}$ of hydrothermally synthesized MIL-53 after calcination and as synthesized [EMIM][Hbdc] derived is-MIL-53.

MAS-NMR-spectroscopy and powder X-ray diffraction, respectively, that is-MIL-53 is stabilized in the ht-form. This stabilization can thus not only be due to enhanced hydrophobicity, as claimed by Liu et al. for a similar material synthesized by an ionothermal approach. ${ }^{5}$ This could only explain the absence of a phase transformation in the presence of a suitable sorbent. The results of the here reported hydrogen sorption experiments also prove the suppression of the purely temperature induced breathing effect, known for MIL-53 type materials.

With [EMIM][Hbdc], a new versatile linker precursor for terephthalate based metal-organic frameworks was synthesized and was successfully employed in an alternative procedure for the synthesis of an MIL-53(Al)-type MOF (see Fig. 4). The completely different characteristics of this isMIL-53 with respect to phase transformation and sorption behavior, in comparison to hydrothermally synthesized MIL-53 (Al) clearly demonstrates the tremendous influence of variations of the reagents used for the syntheses of metal-organic framework materials.

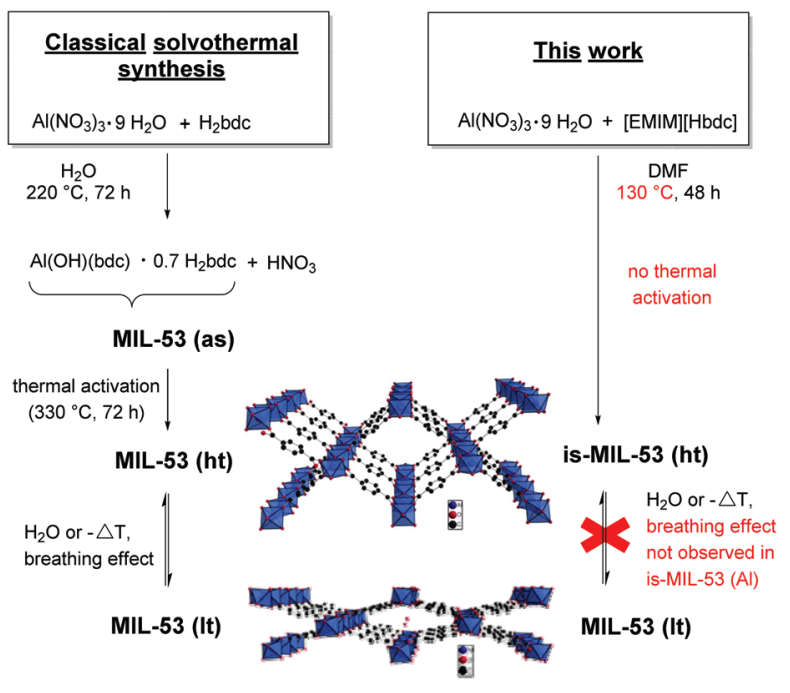

Fig. 4 Comparison of solvothermal MIL-53 synthesis with the route applied in this work. Differences found for is-MIL-53 are marked red. 


\section{Acknowledgements}

Financial support by the "Deutsche Forschungsgemeinschaft" (DFG) within the priority program SPP 1708 and by the "Bavarian Hydrogen Center" (BHC) is gratefully acknowledged.

\section{Notes and references}

$\$$ Experimental details of the synthesis of is-MIL-53 and additional characterization results are provided in the ESI. $\dagger$

1 M. Hartmann, S. Kunz, D. Himsl, O. Tangermann, S. Ernst and A. Wagener, Langmuir, 2008, 24, 8634.

2 A. Schaate, P. Roy, A. Godt, J. Lippke, F. Waltz, M. Wiebcke and P. Behrens, Chem. - Eur. J., 2011, 17, 6643.

3 A. S. Münch, J. Seidel, A. Obst, E. Weber and F. O. R. L. Mertens, Chem. - Eur. J., 2011, 17, 10958.

4 C. Volkringer, D. Popov, T. Loiseau, G. Férey, M. Burghammer, C. Riekel, M. Haouas and F. Taulelle, Chem. Mater., 2009, 21, 5695.
5 J. Liu, F. Zhang, X. Zou, G. Yu, N. Zhao, S. Fan and G. Zhu, Chem. Commun., 2013, 49, 7430.

6 E. R. Parnham and R. E. Morris, Acc. Chem. Res., 2007, 40, 1005.

7 K. Massonne, M. Siemer, W. Mormann and P. Frank, Patent, Process for preparing esters and organic halides, Basf Se, EP 2532646A1, 2012.

8 T. Loiseau, C. Serre, C. Huguenard, G. Fink, F. Taulelle, M. Henry, T. Bataille and G. Férey, Chem. - Eur. J., 2004, 10, 1373.

9 T. Isobe, T. Watanabe, J. B. d'Espinose de la Caillerie, A. P. Legrand and D. Massiot, J. Colloid Interface Sci., 2003, 261, 320 .

10 I. Bezverkhyy, G. Ortiz, G. Chaplais, C. Marichal, G. Weber and J.-P. Bellat, Microporous Mesoporous Mater., 2014, 183, 156.

11 M. Mendt, B. Jee, N. Stock, T. Ahnfeldt, M. Hartmann, D. Himsl and A. Pöppl, J. Phys. Chem. C, 2010, 114, 19443.

12 K. S. Walton and R. Q. Snurr, J. Am. Chem. Soc., 2007, 129, 8552. 